\title{
A Method for Estimating Wall Friction in Turbulent Boundary Layers
}

\author{
Anthony Kendall ${ }^{*}$ and Manoochehr Koochesfahani ${ }^{\dagger}$ \\ Department of Mechanical Engineering, Michigan State University, East Lansing, MI 48824
}

\begin{abstract}
We describe a simple method for estimating wall friction which uses the fit of velocity data to the boundary layer profile in wall units all the way from the wall to the end of the log region. Two different models for the boundary layer profile are examined, the power-series interpolation scheme of Spalding and the Musker profile which is based on the eddy viscosity concept. The accuracy of the new method is quantified using the zero pressure gradient 2-D turbulent boundary layer data of Österlund for which independent measurement of wall shear is available. Results show that the new procedure can provide accurate estimates of wall shear with a mean error of about two percent or better. The usefulness of this method is in its ability to provide accurate estimates of wall shear not only based on full velocity profile data but also sparse data points in the velocity profile, including only a single data point.
\end{abstract}

\section{Introduction}

A common approach for estimating the wall shear stress, $\tau_{w}$, in turbulent boundary layers is the Clauser method $^{1,2}$. In this method, measurement of the mean velocity profile $U(y)$ is used to estimate the friction velocity $u_{\tau} \equiv \sqrt{\tau_{w} / \rho}$ using the fit of the measured data to the logarithmic region of the boundary layer, given by

$$
\frac{U(y)}{u_{\tau}}=\frac{1}{\kappa} \ln \left(\frac{y u_{\tau}}{v}\right)+B .
$$

There are several aspects in the implementation of this method that can introduce uncertainties in the estimated shear stress. One need to select the beginning and ending points within the data set that are believed to fall within the loglinear region, and this brings an element of user subjectivity into the result. A more important aspect is the fact that the estimate of friction velocity is directly related to the choice for the von Kármán constant $\kappa$. Different values of this constant are often reported in the literature ${ }^{3}$, and a number of studies have indicated that the Clauser method produces artificially high friction velocities, especially at the low range of Reynolds numbers ${ }^{4-8}$. For example, the friction velocity estimated from the Clauser method was reported to have an error varying between $8 \%-20 \%$ over a Reynolds numbers in the range of $\operatorname{Re}_{\theta}=1,000-10,000^{5}$.

Measurements of turbulent boundary layer profile quite often contain velocity data much closer to the wall than the logarithmic region, sometimes extending well into the linear sublayer. It would seem one should take advantage of all the velocity data available for estimating wall shear and not rely only on the data in the log-linear region. Advances in microsensors have led to flush surface-mounted optical probes that provide single-point samples of the velocity very close to the wall (e.g. about $70 \mu \mathrm{m}$ from the wall) ${ }^{9,10}$, with the idea of measuring wall shear using velocity data in the linear sublayer. In a typical measurement, however, the actual location in wall units of the velocity information is not known in advance and, depending on the flow Reynolds, it can occur anywhere in the linear sublayer, in the logarithmic region, or in the buffer layer joining these two regions.

Motivated by the issues described in the preceding paragraph, we describe a simple method for estimating wall shear which takes advantage of the fit of the measured velocity data to the boundary layer profile in wall units all the way from the wall to the end of the log region. We assess the performance of the method using recent experimental

\footnotetext{
* Graduate Student.

${ }^{\dagger}$ Professor, AIAA Associate Fellow, Email: koochesf@egr.msu.edu.
} 
data in zero pressure gradient flat-plate turbulent boundary layers for which independent measurements of wall shear are also available. We will show that the new procedure provides accurate estimates of the wall shear with a mean error of about two percent or better. The method is more robust than the Clauser method and it does not require careful selection of data points that are believed to fall into the log-linear region. In addition, the new method is able to provide accurate estimates of wall shear with very sparse data points in the velocity profile, including only a single data point.

\section{Wall Shear Estimation Method and Experimental Data}

\section{A. Experimental Data Base}

The velocity measurements of Österlund ${ }^{11,12}$ are used for assessing the performance of the method we describe in this paper. This data base consists of 70 mean velocity profiles of a zero pressure gradient 2-D turbulent boundary layer measured with hot-wire anemometry at different freestream velocities and downstream locations along a flatplate, covering a Reynolds number in the range 2,533< $\operatorname{Re}_{\theta}<27,320$. For each case, independent measurement of wall shear $\tau_{w}$ is available based on oil-film interferometry. The accuracy of wall shear data is reported to be $1 \%$ (corresponding to $0.5 \%$ accuracy in friction velocity $u_{\tau}$ ).

\section{B. Mean Boundary Layer Profiles}

Two models of turbulent boundary layer mean velocity profile are used in this work. The first is the Spalding profile ${ }^{13}$ which is a power-series interpolation scheme joining the linear sublayer to the logarithmic region. The Spalding profile has the following form

$$
y^{+}=u^{+}+\exp (-\kappa B)\left[\exp \left(\kappa u^{+}\right)-1-\kappa u^{+}-\frac{\left(\kappa u^{+}\right)^{2}}{2}-\frac{\left(\kappa u^{+}\right)^{3}}{6}\right],
$$

where the usual inner variable normalization is used, i.e. $y^{+}=\left(y-y_{0}\right) u_{\tau} / v$ and $u^{+}=U / u_{\tau}$. In these expressions, $U$ is the mean velocity, $v$ is the fluid kinematic viscosity and $y_{0}$ is retained as the wall coordinate to allow for the possibility of uncertainty in wall location. Throughout this work, we use a fix set of boundary layer constants $(\kappa=$ $0.41, B=5.0)$.

The second profile is that due to Musker ${ }^{14}$ and it was suggested to us by Professor Donald Coles of Caltech. The Musker profile is based on the eddy viscosity model and is given by the solution to the following implicit relation

$$
\frac{d u^{+}}{d y^{+}}=\frac{\frac{\left(y^{+}\right)^{2}}{\kappa}+\frac{1}{s}}{\left(y^{+}\right)^{3}+\frac{\left(y^{+}\right)^{2}}{\kappa}+\frac{1}{s}} .
$$

The variable $s$ is solved for by matching the solution $u^{+}\left(y^{+}\right)$from the above equation to the log-linear region (i.e. Equation 1) at sufficiently large $y^{+}$. Using an iterative procedure, for the selected boundary layer constants $(\kappa=0.41, B=5.0)$ we find $s=0.001093$, consistent with previous results in Musker ${ }^{14}$.

The Spalding and Musker profiles are plotted in Figure 1 along with two of the measured velocity profiles in the Österlund data base. By construction, both Spalding and Musker profiles agree in the linear sublayer and logarithmic regions. They differ slightly, however, in the transition between these two regions. Note that the Musker profile provides a better fit to the two measured profiles shown here. One of the measured profiles begins to diverge from the loglinear profile at approximately $y^{+}>300$, depicting the wake component of that profile.

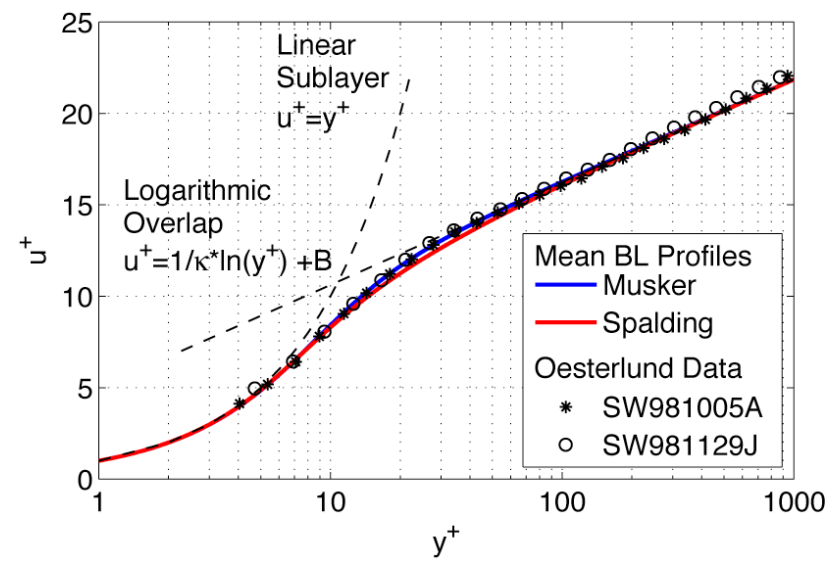

Figure 1. Comparison of Musker and Spalding profiles $(\kappa=0.41, B=5)$ with two profiles from the Österlund data set. 


\section{Friction Velocity Estimation Procedure}

For a given measured data set, the velocity points $U(y)$ in real units are normalized into wall units $u^{+}\left(y^{+}\right)$by selecting the value of friction velocity $u_{\tau}$ and wall location $y_{0}$ that best fit the normalized data against the model profile (i.e. either Spalding or Musker profile). This process is illustrated in Figure 2 for a particular measured data set (Österlund data set SW981005A). In this case, $y_{0}$ was set to zero since the Österlund data base is already referenced to the actual wall location. Figure 2 shows the normalized data in wall units for different iterations on the guess for the friction velocity until the normalized data collapse onto the Musker profile. The iteration sequence was $u_{\tau}=0.5,1.0,0.71,0.79$, and 0.75. For reference, the directly measured value of friction velocity was $u_{\tau} \approx 0.749$ for this case. It can be noted in this figure that an error in the $u_{\tau}$ estimate by less

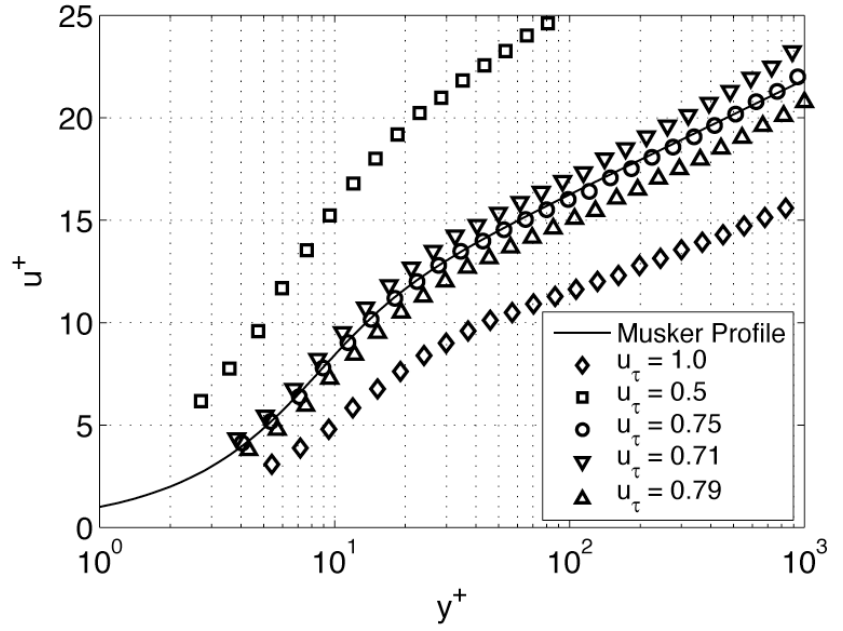

Figure 2. Illustration showing the iterative determination of friction velocity for a measured profile (Österlund data set SW981005A). than $5 \%$ is very easily discernible to visual inspection.

The process just described can, of course, be automated by minimizing the difference between the normalized measured data and the model profile. Two different residual functions defining this difference were considered as defined below.

$$
\begin{aligned}
& \Phi_{1}=\frac{1}{N} \sum_{i=0}^{N} \sqrt{\left(u_{i}^{+}(\text {data })-u_{i}^{+}(\text {model })\right)^{2}} \\
& \Phi_{2}=\frac{1}{N} \sum_{i=0}^{N} \frac{\mid u_{i}^{+}(\text {data })-u_{i}^{+}(\text {model }) \mid}{u_{i}^{+}(\text {model })} .
\end{aligned}
$$

In these expressions, $u_{i}^{+}($data $)$corresponds to the $N$ measured velocity points in wall units located at $y_{i}^{+}$, and $u_{i}^{+}$(model) refers to the model velocities (from Spalding or Musker profile) at those locations. The index $i$ runs from 0 to $N$ because the point $\left(y_{0}^{+}=0, u_{0}^{+}=0\right)$ was added in order to enforce the no-slip boundary condition. The residual function $\Phi_{2}$, which is defined based on a fractional difference between points on the data and model profiles, was found to offer an advantage as will be described later.

A two-parameter optimization routine was written in MATLAB to determine the set of $u_{\tau}$ and $y_{0}$ that minimize the residual function for each of the measured profiles in the Österlund data set. The measured data are provided in real units, along with an initial user-specified search domain in the two parameter space. The search domain is iteratively refined until $u_{\tau}$ and $y_{0}$ converge to within a userdefined tolerance level. In the results presented here, the tolerance levels were selected to be $0.0001 \mathrm{~m} / \mathrm{s}$ for $u_{\tau}$ (better than $0.05 \%$ for all cases) and $10 \mu \mathrm{m}$ for $y_{0}$ for cases where wall location was considered variable. Figure 3 shows an example of a shaded contour plot of the residual function obtained with this process for one particular data set. Note that it is a smooth surface with a well-defined global minimum corresponding to the values of $u_{\tau}$ and $y_{0}$ that provide

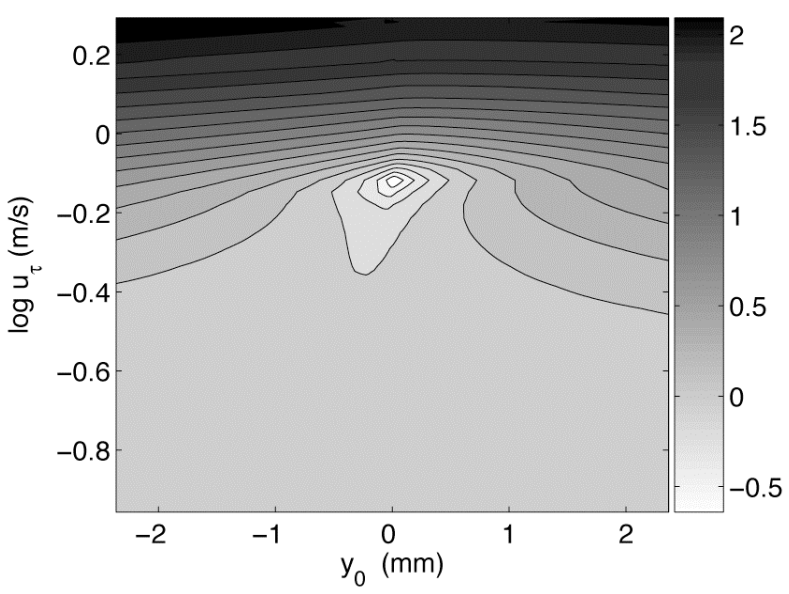

Figure 3. Shaded contour map of the log of residual $\Phi_{1}$ versus $u_{\tau}$ and $y_{0}$ for the fit of a measured profile (Österlund data set SW981005A) to Musker profile. 
the best fit to the model profile. We further note that the residual function is less sensitive to $y_{0}$ variation than to $u_{\tau}$ (note the abscissa is logarithmic). Thus, convergence in $y_{0}$ is generally slower than that in $u_{\tau}$. For each data set, the accuracy of the estimated friction velocity $u_{\tau}$ (estimated) from the optimization routine is evaluated by its comparison with the actual measured value $u_{\tau}$ (measured) from oil-film interferometry. The resulting percentage error defined by $\delta_{u \tau}=\left(u_{\tau}\right.$ (estimated $)-u_{\tau}$ (measured $\left.)\right) / u_{\tau}$ (measured) provides an ensemble of 70 samples corresponding to the 70 velocity profiles in the Österlund data base. The mean and rms errors of this ensemble form the basis of our discussion in the next section.

\section{Results and Discussion}

\section{A. Friction Factor Estimate Based on Full Profile}

We will first consider the situation where all the data from a measured velocity profile are available for estimating the friction velocity. One parameter that needs to be selected is the largest $y^{+}$value, $y_{\max }^{+}$, that should be used in the optimization process (i.e. only data points with $y_{i}^{+} \leq y_{\max }^{+}$will be included in the optimization.). Because $u_{\tau}$ is not known prior to optimization, we estimated the optimum $u_{\tau}$ across a range of $y_{\max }^{+}$values and then sorted out the results for presentation.

Figure 4 depicts a histogram of error in the friction factor estimate, $\delta_{u \tau}$, for all 70 Österlund data sets based on the fit to the Musker profile and the $y_{\max }^{+}$cutoff set to $y_{\max }^{+}=500$. As before, $y_{0}$ was set to zero since the Österlund data base is already referenced to the actual wall location. The important result is that the errors all fall between $-1.5 \%$ and $1.75 \%$ regardless of $\operatorname{Re}_{\theta}$ value of the data. The estimation of friction velocity in this case has a mean error of about $-0.25 \%$ and an rms error of about $0.75 \%$.

We now compare the performance of the two model profiles of Musker and Spalding as a function of the $y_{\max }^{+}$cutoff used in the optimization. Results are presented in Figure 5 in terms of the mean and rms error in the estimated friction velocity. For this comparison the residual function $\Phi_{1}$ was used and $y_{0}$ was set to zero. We note that the Musker profile generally produces a lower mean error overall and is significantly better in the range $20 \leq y_{\max }^{+} \leq 100$. This range of $y^{+}$values corresponds to the transition region between the linear sublayer and the log-linear region. It was already noted in Figure 1 that Musker profile seemed to fit the data more closely in this region than Spalding profile. Because of this better performance, Musker profile is used to obtain the results presented from here on. We note the

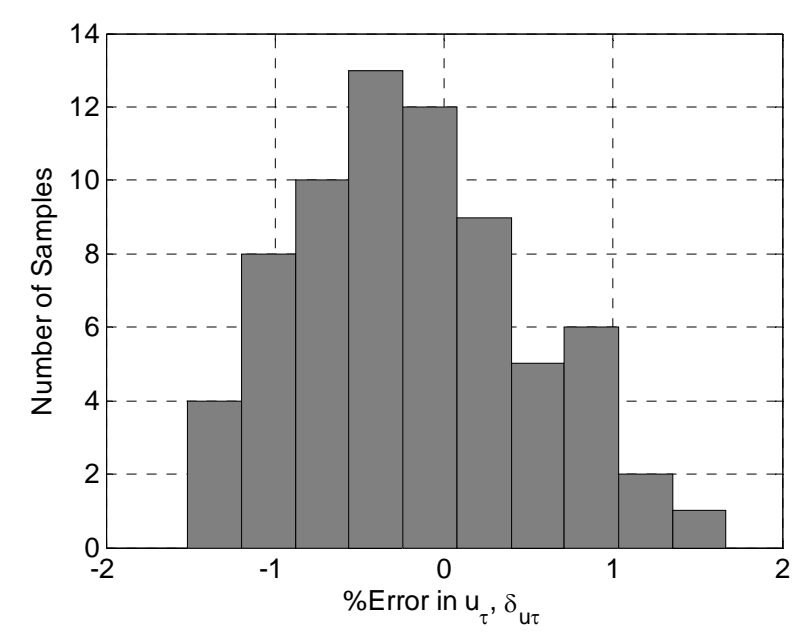

Figure 4. Histogram of $\%$ error in friction velocity for all Österlund data sets using the Musker profile and $\Phi_{2}$ ( $y_{0}$ fixed at zero, $\mathrm{y}_{\max }^{+}=500$ ).

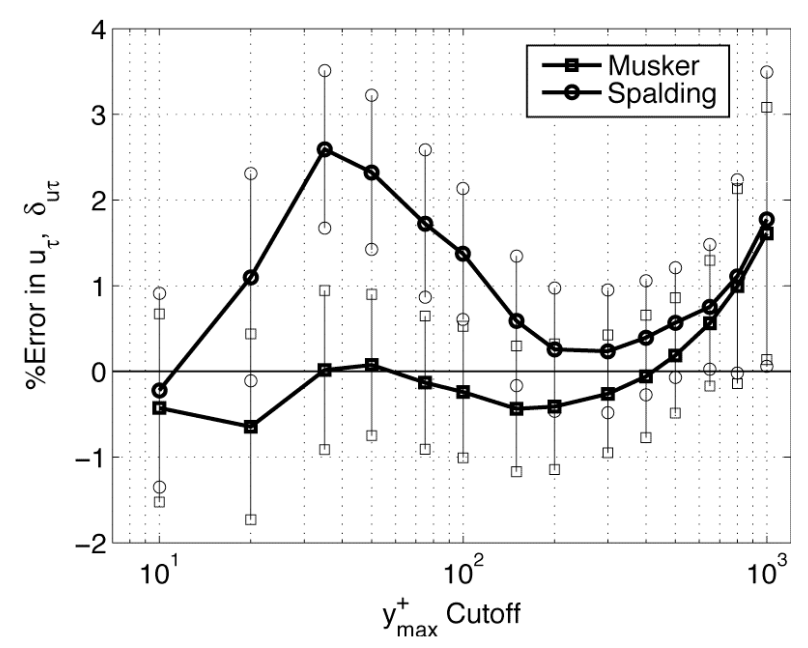

Figure 5. Comparison of the performance of Musker and Spalding profiles based on the mean and $\mathbf{r m s}$ of $\%$ error in friction velocity ( $y_{0}$ fixed at zero, residual function $\Phi_{1}$ ). 
remarkable accuracy with which the friction factor is estimated over a large range of $y_{\max }^{+}$cutoff values, with a mean error that is better than $1 \%$ (or $2 \%$ for wall shear).

Figure 5 also illustrates that the mean error starts to increase noticeably at large values of $y_{\max }^{+}$cutoff, an effect that is caused by the data points in the measurement that fall in the wake component of the boundary layer, whose deviations from the model profile lead to residuals that are large in magnitude. This detrimental influence can be reduced by defining a different residual function $\Phi_{2}$ that is based on the fractional difference between points on the data and model profiles (see Equation 5). Results based on the minimization of $\Phi_{2}$ are shown in Figure 6 in comparison with those based on $\Phi_{1}$. It can be seen that the effect of the velocity measurements at large values of $y^{+}$is significantly reduced with a net effect that the friction factor can be estimated more accurately over a much larger range of $y_{\max }^{+}$cutoff values, well after the wake component starts to deviate from the logarithmic region.

In all the results discussed so far $y_{0}$ was fixed at zero since the Österlund data base is already referenced to the actual wall location (known to $\pm 5 \mu \mathrm{m}$ ). The ability of the optimization routine to recover non-zero wall locations was tested by deliberately offsetting several of the Österlund data sets in the $y$-direction by $40 \mu \mathrm{m}$. The optimization routine recovered this offset to an accuracy of approximately $\pm 2 \mu \mathrm{m}$ in the range $100<y_{\max }^{+}<1000$ (in this case, the tolerance for determining $y_{0}$ was lowered to $1 \mu \mathrm{m}$ ). The optimization routine was performed a second time on the entire Österlund data base, allowing for non-zero $y_{0}$. The mean wall location that was recovered was within the \pm 5 $\mu \mathrm{m}$ accuracy of the reported actual wall location. Allowing for the possibility of a non-zero wall location in the search routine had only a minor influence on the estimated values of friction factor (about $0.25 \%$ ).

\section{B. Friction Factor Estimate Based on Sparse Data (1, 2, and 3 Point Data)}

We now discuss the accuracy of friction velocity estimates based on very sparse data. In particular, we are interested in the case where there is only one mean velocity data point measured at an arbitrary location away from the wall. This, for example, would be the case for the optical probe described in References 9, 10 , which is designed to obtain single-point velocity data at about $70 \mu \mathrm{m}$ away from the wall.

Single-point and multi-point data were extracted from the Österlund data set in the following manner. A nominal $y$ location was first chosen in real units for the single-point case (e.g. $y_{1}=50 \mu \mathrm{m}, 75 \mu \mathrm{m}, 100 \mu \mathrm{m}$, etc.) For two- and three-point cases, the locations of the additional velocity data were arbitrarily selected to be at $y_{2}=2 y_{1}$ and $y_{3}=2 y_{2}$, simulating a hypothetical two- or three-probe measurement device. Since the measured data sets do not necessarily contain data at these exact locations, instead of interpolating the data, we chose to select the nearest data points to these nominal locations. The criterion used was that if there were no data points within $10 \%$ of the nominal $y$ -

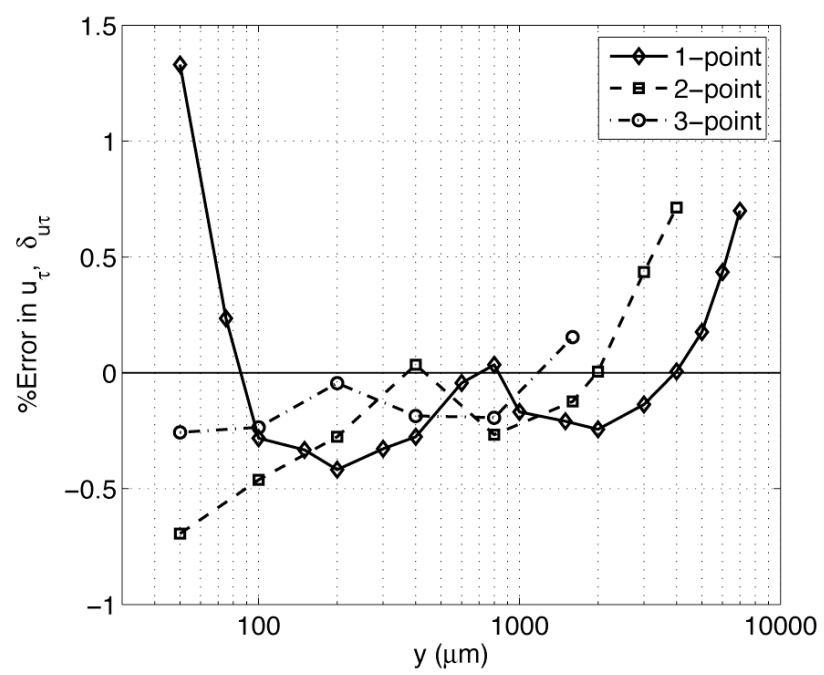

Figure 7. Mean \% error in friction velocity estimated from sparse data (Musker profile, $y_{0}$ fixed at zero). Note that $y$ locations are in physical units. 
locations, that data set was excluded from the analysis. The spatial density of data in the Österlund data base is high enough that only a few cases were excluded from this analysis. The exception was the lowest location $y_{1}=50 \mu \mathrm{m}$, for which less than half of the data sets were actually used.

Figure 7 shows the mean $\%$ error in the friction velocity estimated based on sparse data. The most important observation is that we can obtain an accurate estimate of friction velocity with only a single data point. The corresponding values of mean error are comparable to those obtained earlier based on the full-profile data. Adding second and third velocity points helps improve the mean error primarily for the case closest to the wall. In all cases, the rms error was about $1.5 \%$ (not shown in this figure). Note, however, that two-point data is the minimum requirement for determining both friction velocity and wall location $y_{0}$.

\section{Conclusion}

The results show that accurate estimates of friction velocity can be obtained from the new method we have described. The mean accuracy is within $1 \%$ for the friction velocity, corresponding to $2 \%$ for wall friction. A particular advantage of this method is that it can be used with velocity data that do not necessarily fall in the logarithmic region nor in the linear sublayer. As a result, the procedure can be easily automated without user intervention. Furthermore, its accuracy is equally good when using a single mean velocity data point.

We expect this approach to be useful for the estimation of wall friction in any turbulent boundary layer in which the profile in wall units collapses onto a common universal curve, e.g. that represented by the Musker profile. This includes turbulent boundary layers under favorable pressure gradient and those in adverse pressure gradient not too close to the separation point. Preliminary application of this method to the favorable pressure gradient superpipe boundary layer data ${ }^{15,16}$ shows promising results.

\section{Acknowledgments}

We thank Professor Hassan Nagib for providing us with the Österlund data set. The authors are grateful to Professor Donald Coles for discussions and many helpful suggestions.

\section{References}

${ }^{1}$ Clauser, F. H., "The Turbulent Boundary Layer," Advances in Applied Mechanics, 4, 1956, pp. 1-51.

${ }^{2}$ Fernholz , H. H. and Finley, P. J., "The Incompressible Zero-Pressure-Gradient Turbulent Boundary Layer: an Assessment of the Data," Prog. Aerospace Science, 32, 1996, pp. 245-311.

${ }^{3}$ Zanoun, E., Durst, F., and Nagib, H., "Evaluating the Law of the Wall in Two-Dimensional Fully Developed Channel Flows," Phys. Fluids, 15, 2003, pp. 3079-3089.

${ }^{4}$ Kline, S. J., Reynolds, W. C., Schraub, F. A., and Runstadler, P.W., "The Structure of Turbulent Boundary Layers,” J. Fluid Mech., 30, 1967, pp. 741-773.

${ }^{5}$ Blackwelder, R. and Haritonidis, J., "Scaling of the Bursting Frequency in Turbulent Boundary Layers," J. Fluid Mech., 132, 1983, pp. 87-103.

${ }^{6}$ Spalart, P., "Direct Simulation of a Turbulent Boundary Layer up to $\operatorname{Re}_{\theta}=1410$," J. Fluid Mech., 187, 1988, pp. 61-98.

${ }^{7}$ Ong, L. and and Wallace, J. M., "Joint Probability Density Analysis of the Structure and Dynamics of the Vorticity Field of a Turbulent Boundary Layer,” J. Fluid Mech., 367, 1998, pp. 291-328.

${ }^{8}$ Wei, T., Schmidt, R., and McMurtry, P., "Comment on the Clauser Chart Method for Determining the Friction Velocity," Exp in Fluids, 38, 2005, pp. 695-699.

${ }^{9}$ Fourguette, D., Modarress, D., Taugwalder, F, Wilson, D, Koochesfahani, M. and Gharib, M., "Miniature and MOEMS flow sensors," AIAA Paper No. AIAA-2001-2982.

${ }^{10}$ Fourguette, D., Modarress, D., Wilson, D., Koochesfahani, M. and Gharib, M., "An Optical MEMS-Based Shear Stress Sensor for High Reynolds Number Applications,” AIAA Paper No. AIAA-2003-0742.

${ }^{11}$ Österlund, J. M., "Experimental Studies of Zero Pressure-Gradient Turbulent Boundary-Layer Flow," Ph.D. thesis, Department of Mechanics, Royal Institute of Technology, Stockholm, 1999.

${ }^{12}$ Österlund, J. M., Johansson, A.V., Nagib, H. M., and Hites, M. H., “A Note on the Overlap Region in Turbulent Boundary Layers," Phys. Fluids, 12, 2000, pp. 1-4.

${ }^{13}$ Spalding, D.B., "A Single Formula for the Law of the Wall," J. Applied Mechanics, Transaction of the ASME, Series E., Vol. 83, 1961, p. 455.

${ }^{14}$ Musker, A. J., "Explicit Expression For the Smooth Wall Velocity Distribution in a Turbulent Boundary Layer," AIAA J., 17, 1979, pp. 655-657.

${ }^{15}$ Zagarola, M. V., "Mean Flow Scaling of Turbulent Pipe Flow," Ph.D. Thesis, Department of Mechanical and Aerospace Engineering, Princeton University, 1996.

${ }^{16}$ McKeon, B. J., Li, J., Jiang, W., Morrison, J. F., and Smits, A. J., "Further Observations on the Mean Velocity Distribution in Fully Developed Pipe Flow," J. Fluid Mech., 501, 2004, pp. 135-147. 